\title{
Molecular characterization and expression analysis of pearl millet plasma membrane proteolipid 3 (Pmp3) genes in response to abiotic stress conditions
}

\author{
Richa K. Yeshvekar ${ }^{\mathrm{b}}$, Rahul B. Nitnavare ${ }^{\mathrm{b}}$, Thammineni Chakradhar ${ }^{\mathrm{a}}$, Pooja Bhatnagar-Mathur ${ }^{\mathrm{b}}$, \\ Malireddy K. Reddy ${ }^{\mathrm{a}}$, Palakolanu Sudhakar Reddy ${ }^{\mathrm{a}, \mathrm{b}}$,* \\ a Plant Molecular Biology Group, International Centre for Genetic Engineering and Biotechnology (ICGEB), Aruna Asaf Ali Marg, New Delhi 110 067, India \\ b International Crops Research Institute for the Semi-Arid Tropics (ICRISAT), Patancheru, Hyderabad 502 324, Telangana, India
}

\section{A R T I C L E I N F O}

\section{Keywords:}

Pennisetum glaucum

Plasma membrane proteolipid 3 (Pmp3)

Low temperature

Abiotic stress

Gene expression

Network analysis

\begin{abstract}
A B S T R A C T
Plasma membrane proteolipid 3 (Pmp3) is a gene family involved in abiotic stress response and cellular protection. Here we report cloning of two genes PgPmp3-1 and PgPmp3-2 from Pennisetum glaucum, and characterization with respect to their functions and responsiveness to various abiotic stresses. Both PgPmp3-1 and PgPmp3-2 genes are 171 bp long and encode for 56 amino acid long peptides. PgPmp3 sequences share 70-99\% sequence identity with their homologs. Protein secondary structure prediction revealed membranespanning regions containing a membrane potential modulator domain in both PgPmp3 proteins. In silico network analyses revealed Pmp3 co-expression and association with proteins conferring abiotic stress tolerance in plants. Expression profiles of PgPmp3-1 and PgPmp3-2 revealed their up-regulation in $P$. glaucum under cold and salt stresses, but showed reduced expression in response to heat stress. These findings provide insight into the role of P. glaucum Pmp3 in abiotic stress amelioration.
\end{abstract}

\section{Introduction}

Crop productivity is adversely affected primarily by abiotic stress conditions such as drought, salinity and extreme high or low temperatures. To withstand abiotic stress, plants alter their physiological, biochemical and molecular processes and successfully adapt to stressful environments (Islam and Tuteja, 2012). Pennisetum glaucum, commonly known as pearl millet, is a hardy and robust crop found in arid and semi-arid regions of India and Africa. It often encounters abiotic stress conditions such as drought, extreme temperatures, high salinity and low pH of soil. High salinity and low temperature stresses during seed germination and seed setting prove detrimental to the overall yield of $P$. glaucum. Several studies have elucidated the effect of environmental stresses on expression of various genes and proteins in this dry land cereal crop (Reddy et al., 2012, 2014, 2015). Nevertheless, studies regarding response of various genes and their respective roles in alleviating the stress conditions, especially extreme temperatures and high salinity, might provide additional insights into $P$. glaucum stress adaptation.

The initial effect of abiotic stresses is perceived on the cell wall and plasma membrane that act as an interface between the cell and external environment (Panjabi-Sabharwal et al., 2010). Plasma membrane plays an important role in keeping the cell intact, maintaining cellular osmosis and signal transduction. Exposure to abiotic stresses increases the membrane permeability thereby allowing loss of electrolytes through the cell (Lyons, 2012). Thus, protection of plasma membrane is important during exposure to abiotic stress conditions. Some integral membrane proteins play important roles in cell-cell interaction, ion transport and signal transduction (Marmagne et al., 2004). Proteins such as gated aquaporins (plasma membrane intrinsic proteins), $\mathrm{H}^{+}$ ATPase, receptor protein kinases and calmodulin, which are present on plasma membrane, contribute to stress tolerance in plants (Arazi et al., 1999; Li et al., 2015; Roy et al., 2005; Osakabe et al., 2010).

Plasma membrane proteolipid 3 (Pmp3), first reported in Saccharomyces cerevisiae, is a gene involved in combating low temperature and salt stress induced membrane instability (Navarre and Goffeau, 2000). The homologs of Pmp3, also known as rare cold inducible (RCI) or low temperature inducible (LTI) genes (Medina et al., 2001; ChangQing et al., 2008) have been shown to express under abiotic stress conditions in several plant species such as Arabidopsis thaliana, Triticum aestivum, Oryza sativa, Zea mays and Hordeum vulgare (Medina et al., 2001; Khurana et al., 2015; Chang-Qing et al., 2008; Goddard et al.,

\footnotetext{
Abbreviations: Pmp3, plasma membrane proteolipid 3; qPCR, quantitative real-time PCR; RCI, rare cold induced; LTI, low temperature induced

* Corresponding author at: Cell, Molecular Biology \& Genetic Engineering Group, Research Program - Genetic Gains, International Crops Research Institute for the Semi-Arid Tropics (ICRISAT), Patancheru, 502324 Hyderabad, Telangana, India.

E-mail address: p.sudhakarreddy@cgiar.org (P.S. Reddy).
} 
1993). Pmp3 encode for highly hydrophobic proteins that are embedded in plasma membrane with two putative transmembrane domains and small extracellular and cytoplasmic regions. Pmp3 protein is known to modulate plasma membrane potential to maintain cellular ion homeostasis and helps in survival during salt stress (De Block et al., 2015; Serrano and Rodriguez-Navarro, 2001). It is also suggested that Pmp3 regulates a depolarizing cation and proton leak, reducing sensitivity to salinity and low $\mathrm{pH}$ conditions. Individual Pmp3 genes belonging to the same organism could be differentially expressed under various stress conditions, as reported in A. thaliana and the alkali grass, Puccinellia tenuiflora (Medina et al., 2007; Chang-Qing et al., 2008). Although Pmp3 has been reportedly induced in response to salt or cold stress, its exact role is still unclear. Thus, we considered it important to study the function of Pmp3 genes from $P$. glaucum, a highly resilient crop plant grown in dry lands.

In the present study, two genes, PgPmp3-1 and PgPmp3-2 from $P$. glaucum, were cloned and characterized with respect to abiotic stress responsiveness. The quantitative expression of these genes was monitored in response to different abiotic stress conditions such as drought, high salinity, high and low temperatures. To shed some light on the role of Pmp3 genes in stress alleviation, their interaction and co-expression with other proteins was predicted using computational methods.

\section{Materials and methods}

\subsection{Plant material and abiotic stress treatments}

Fourteen days-old seedlings of $P$. glaucum were subjected to different abiotic stress conditions for variable periods, as described earlier (Reddy et al., 2015). Briefly, the seedlings were subjected to drought stress by withholding water for 12 to $72 \mathrm{~h}$. Low and high temperature stress conditions were simulated by incubating the plants at $4{ }^{\circ} \mathrm{C}$ and $45^{\circ} \mathrm{C}$, respectively, for different time intervals, ranging between 0.5 and $10 \mathrm{~h}$. Salinity stress was administered by dipping the seedlings in $250 \mathrm{mM}$ solution of sodium chloride for $1 \mathrm{~h}, 4 \mathrm{~h}, 8 \mathrm{~h}, 12 \mathrm{~h}, 24 \mathrm{~h}$ and $36 \mathrm{~h}$. Control conditions were maintained in greenhouse for each stress treatment. The tissue samples were collected from plants subjected to stress conditions and respective control plants, at different time intervals. The samples were collected in three biological replicates, flash frozen in liquid nitrogen and stored at $-80{ }^{\circ} \mathrm{C}$ for subsequent RNA isolation and transcript analysis.

\subsection{Cloning of the PgPmp3 $c D N A$ and genomic clones}

The stress responsive EST database of $P$. glaucum was searched to find clones showing maximum identity with the Pmp3 genes (PgPmp3-1 GenBank accession no. CD725134 and PgPmp3-2 GenBank accession no. CD724750) (Mishra et al., 2007). The PgPmp3-1 and 3-2 genes were PCR amplified by using cDNA and genomic DNA as templates. $150 \mathrm{ng}$ of PgPmp3-1 forward (5'-ATGTCGGACGGCACGGCGAACT-3) and reverse (5'-CTACTTGGTGATGGCCCAGAC) and PgPmp3-2 forward (5'-ATGTCAGAGGGGACGGCCAACT- $3^{\prime}$ ) and reverse (5'-CTACTTGGTGATGGCGTAGACG-3') gene specific primers along with $200 \mu \mathrm{M}$ of dNTPs, 2.5 units of Taq DNA polymerase (Invitrogen) and genomic DNA/cDNA template in a $50 \mu \mathrm{l}$ reaction volume. The PCR cycling conditions include $94^{\circ} \mathrm{C}$ for $1 \mathrm{~min}, 55^{\circ} \mathrm{C}$ for $1 \mathrm{~min}$ and $72{ }^{\circ} \mathrm{C}$ for 2 min for 30 cycles. Amplified PCR products were cloned into the Topo-TA 4.0 vector (Invitrogen) according to the manufacturer's protocol and sequenced.

\subsection{Sequence analysis of PgPmp3 genes}

The BLASTP and BLASTN programmes from National Centre for Biotechnology Information (NCBI) were used to perform sequence similarity searches for identification of genes encoding Pmp3 from other plant species. Multiple sequence alignments were performed by using ClustalW of MacVector. The PgPmp3 genes were characterized by determining the open reading frame (ORF) length and intron numbers. This was confirmed by comparing the sequences of cDNA and respective genomic clones using the EMBL sequence alignment and MacVector ClustalW programmes. Translated cDNA sequences from other plant species were used to construct a neighbor-joining tree. Theoretical isoelectric point (pI), molecular weight, hydropathy analysis, aliphatic index and estimated half-life of the PgPmp3-1 and PgPmp3-2 proteins were determined using the Expert protein analysis system (EXPASY) tools (http://www.ebi.ac.uk/Tools).

\subsection{Structural and network analysis of Pmp3 proteins}

The secondary structure of Pmp3 proteins was predicted by using PSIPRED (http://bioinf.cs.ucl.ac.uk/psipred/) (Buchan et al., 2013). The interaction of Pmp-3 with other genes and proteins, its derived functions and conserved co-expression was predicted using the Search Tool for the Retrieval of Interacting Genes/Proteins (STRING) (Szklarczyk et al., 2015).

\subsection{RNA isolation, $c D N A$ synthesis and $q P C R$ analysis}

Total RNA was isolated from $P$. glaucum seedlings exposed to different abiotic stress conditions and their corresponding controls using the TRIzol reagent (Invitrogen $\mathrm{GmbH}$, Karlsruhe, Germany). cDNA was synthesized using first strand cDNA synthesis kit (Invitrogen $\mathrm{GmbH}$, Karlsruhe, Germany) and used for qPCR amplification using specific primers (PgPmp3-1 [F: 5'-CGAACTGCATCGACATCATC-3' and R: 5'-GGCAGATCCAGAACTCAACC-3'], PgPmp3-2 [F: 5'-AACTGCGTGGACATCCTGA-3' and R: 5'-GCGTAGACGGCGTAGATGAT-3'] and PgMDH [F: 5'-AGAAGGCGCTTGCTTACTCAT-3' and R: 5'-CAGTTCTGGGTGAGGGAATCT-3']). qPCR reactions were performed in optical 96-well plates with an iCycler (BioRad, USA) using SYBR ${ }^{\circledast}$ Green. The reaction conditions were programmed to $2 \mathrm{~min}$ at $95^{\circ} \mathrm{C}$ (polymerase activation), 40 cycles of $95^{\circ} \mathrm{C}$ for $15 \mathrm{~s}$ and $60^{\circ} \mathrm{C}$ for $1 \mathrm{~min}$. Amplicon dissociation curves were recorded after cycle 40 by heating from $60^{\circ} \mathrm{C}$ to $95^{\circ} \mathrm{C}$ with a ramp speed of $1.9^{\circ} \mathrm{C} \mathrm{min}{ }^{-1}$. Experiments were performed independently three times, and the average data was considered for further analysis. The relative change in expression levels of Pmp3 transcripts in different tissues of the plant or in response to abiotic stress conditions was predicted using REST software (Pfaffl et al., 2002) using PgMDH as the reference gene (Reddy et al., 2015). Statistical analyses were performed using the CoStat version 6.204 (Cohort Software, Monterey, CA, USA), applying the one-way ANOVA test. Means were compared using the Tukey-Kramer; difference regarded statistically significant at $p<0.05$.

\section{Results}

\subsection{Cloning, sequence analysis and genomic organization of the PgPmp3} genes

The sequence similarity searches for identifying $P m p 3$ genes in $P$. glaucum revealed 3 putative $P m p 3$ homologs based on sequences from other plant species. In addition to PgPmp3-1 and PgPmp3-2, an EST CD725521.1 was identified. Upon alignment with Pmp3 gene homologs from other organisms, 20 to $83 \%$ homology was observed with a maximum query cover of $25 \%$ which was much lower than what was achieved with PgPmp3-1 and PgPmp3-2 (70 to 99\% identity with a query cover of 30 to 41\%). The sequence similarity of EST CD725521.1 with yeast Pmp3, PgPmp3-1 and PgPmp3-2 at nucleotide level was lower (66\%, 49\% and $42 \%$ respectively). Considering that these were not significant, we chose to restrict the scope of this study with PgPmp3-1 and PgPmp3-2 only.

The $171 \mathrm{bp}$ long cDNA fragments corresponded to PgPmp3-1 (GenBank accession no. CD725134) and PgPmp3-2 (GenBank accession no. CD724750) respectively. BLASTN indicated that the two genes shared $87 \%$ identity with each other, while exhibiting 70 to $99 \%$ identity with homologous genes from monocots and dicots. The structural organization of PgPmp3-1 and PgPmp3-2 genes was studied by comparative analysis of the genomic and CDS sequences. The 
a Pmp3-1

ATGTCGGACGGCACGGCGAACTGCATCGACATCATCCTCGCCATCATCCTGCCGCCGCTCGGGGTCTTCTTCAAGTTCGG 80

$\begin{array}{lllllllllllllllllllllllllll}M & \mathrm{~S} & \mathrm{D} & \mathrm{G} & \mathrm{T} & \mathrm{A} & \mathrm{N} & \mathrm{C} & \text { I } & \mathrm{D} & \text { I } & \text { I } & \text { L } & \text { A } & \text { I } & \text { I } & \text { L } & \text { P } & \text { P } & \text { L } & G & \text { V } & \text { F } & \text { F } & \text { K } & \text { F } & G\end{array}$

CTGCGGGGTCAGTAAAATCTAACCTCCGTCTCTCTCTCTCCGGTGGTGGTTCAGGGCTCTTTCATTTTCTTGCTGGCGAT C G V

TCGTTTGCAGCTCGCTCGCCGCCGACCGGCCGGCCGGCCGCCGTGCATGATACTGTGCGCCGGTTTTATTTTGTTTGTTT

ACGACTGCTGCTGCTACTGTGTGTAGTAGTGTCTGTGGGCTGATGACAGGTGACTGTCGTTGCTGTGCTTTGCAGGTTGA

GTTCTGGATCTGCCTCGTCCTCACCTTCTTCGGCTACCTCCCCGGCATCATCTACGCCGTCTGGGCCATCACCAAGTAG 399

$\begin{array}{lllllllllllllllllllllllllll} & \text { F } & W & I & C & \text { L } & V & \text { L } & \text { T } & \text { F } & \text { F } & \text { G } & \text { Y } & \text { L } & \text { P } & \text { G } & \text { I } & \text { I } & \text { Y } & \text { A } & \text { V } & \text { W } & \text { A } & \text { I } & \text { T } & K & \text { * }\end{array}$

b Рmp3-2

ATGTCAGAGGGGACGGCCAACTGCGTGGACATCCTGATCGCCATCATCCTGCCGCCGCTGGGGGTGTTCCTCAAGCACGG 80 $\begin{array}{lllllllllllllllllllllllllll}M & S & E & G & T & A & N & C & V & D & I & L & I & A & I & I & L & P & P & L & G & V & F & L & K & H & G\end{array}$

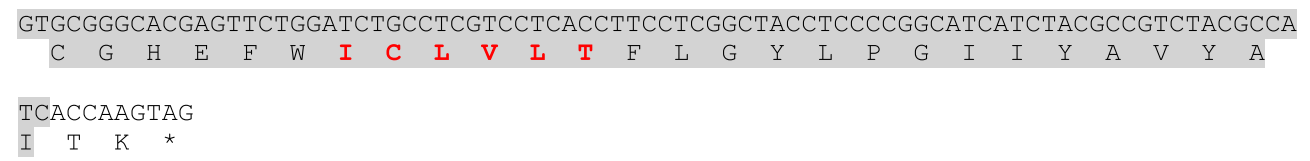

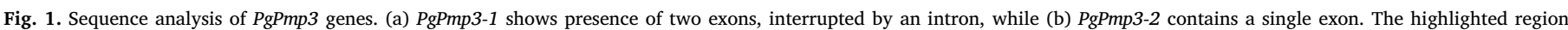
corresponds to the conserved domain encoding plasma membrane potential modulator.

genomic organization indicated presence of two exons of $89 \mathrm{bp}$ and 82 bp length, interrupted by a single 229 bp intron in PgPmp3-1 gene (Fig. 1a), corresponding to a 171 bp coding region. In contrast, PgPmp32 gene contained only a single 171 bp long exon (Fig. 1b).

\subsection{Characteristics of PgPmp3 proteins and phylogenetic analysis}

The two proteins, viz., PgPmp3-1 and PgPmp3-2 were found to be comprised of 56 amino acids each, and their molecular weights were predicted as $6.172 \mathrm{kDa}$ and $6.109 \mathrm{kDa}$ respectively, using the ProtParam tool (Expasy). The theoretical isoelectric points were estimated to be 4.56 and 5.97 respectively. The aliphatic index, a measure of volume occupied by the side chains of the protein was 132.32 for PgPmp3-1, while it was 139.29 for PgPmp3-2. BLASTP indicated that PgPmp3-1 and PgPmp3-2 proteins shared 83\% identity with each other. Proteins exhibiting $>70 \%$ sequence identity with PgPmp3-1 and PgPmp3-2 were identified by using BLASTP analysis. The amino acid sequence alignment showed two conserved domains, IILAIILPPLGV and EFWICL (Fig. 2), specific for the Pmp3 genes (Pfam01679), as confirmed by using NCBI's conserved domain database (Marchler-Bauer et al., 2011). Additionally, a 46 amino acid long proteolipid membrane potential modulator motif was predicted to be present between the $8^{\text {th }}$ and $54^{\text {th }}$ position of the Pmp3 protein.

The sequences of proteins homologous to P. glaucum PgPmp3-1 and

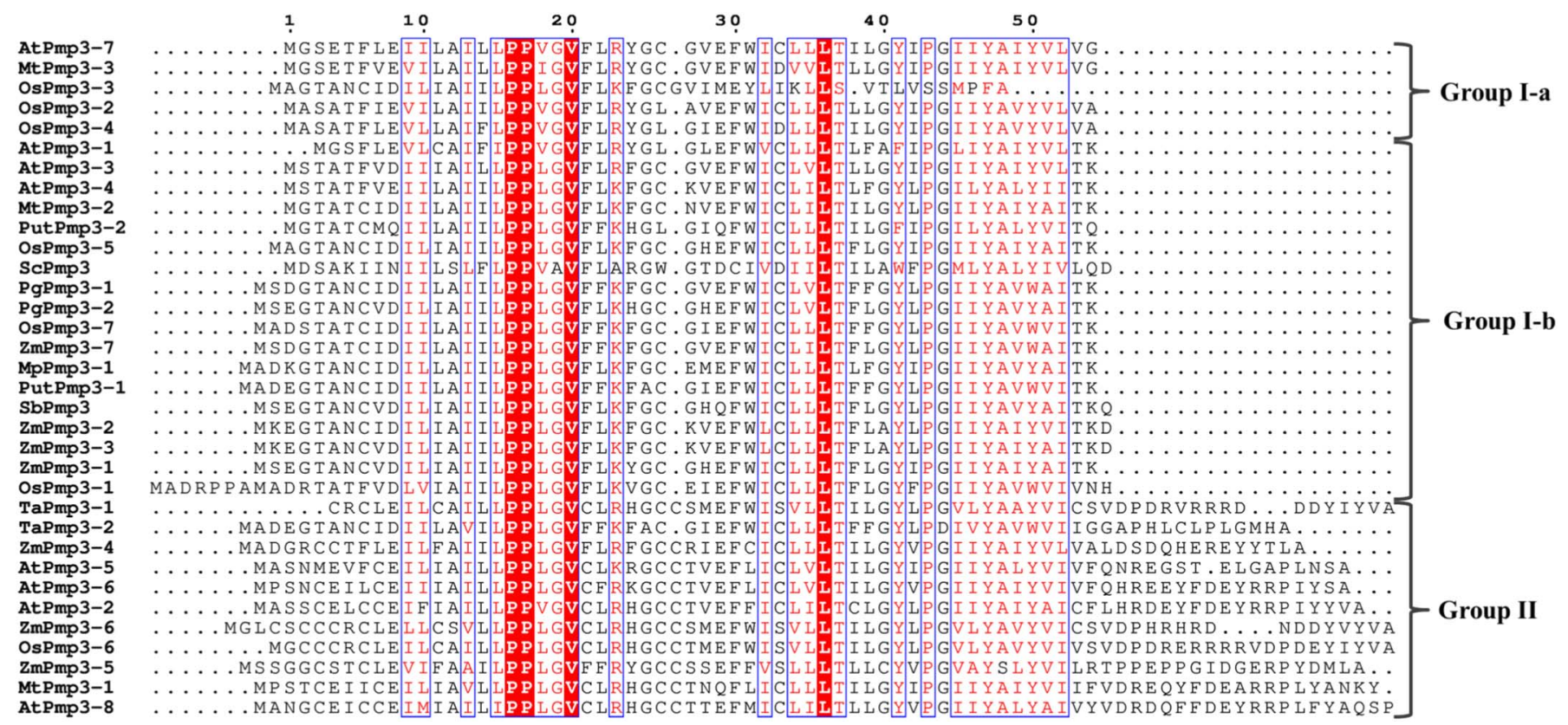

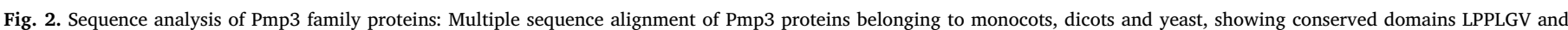
LVLT (displayed in boxes). 


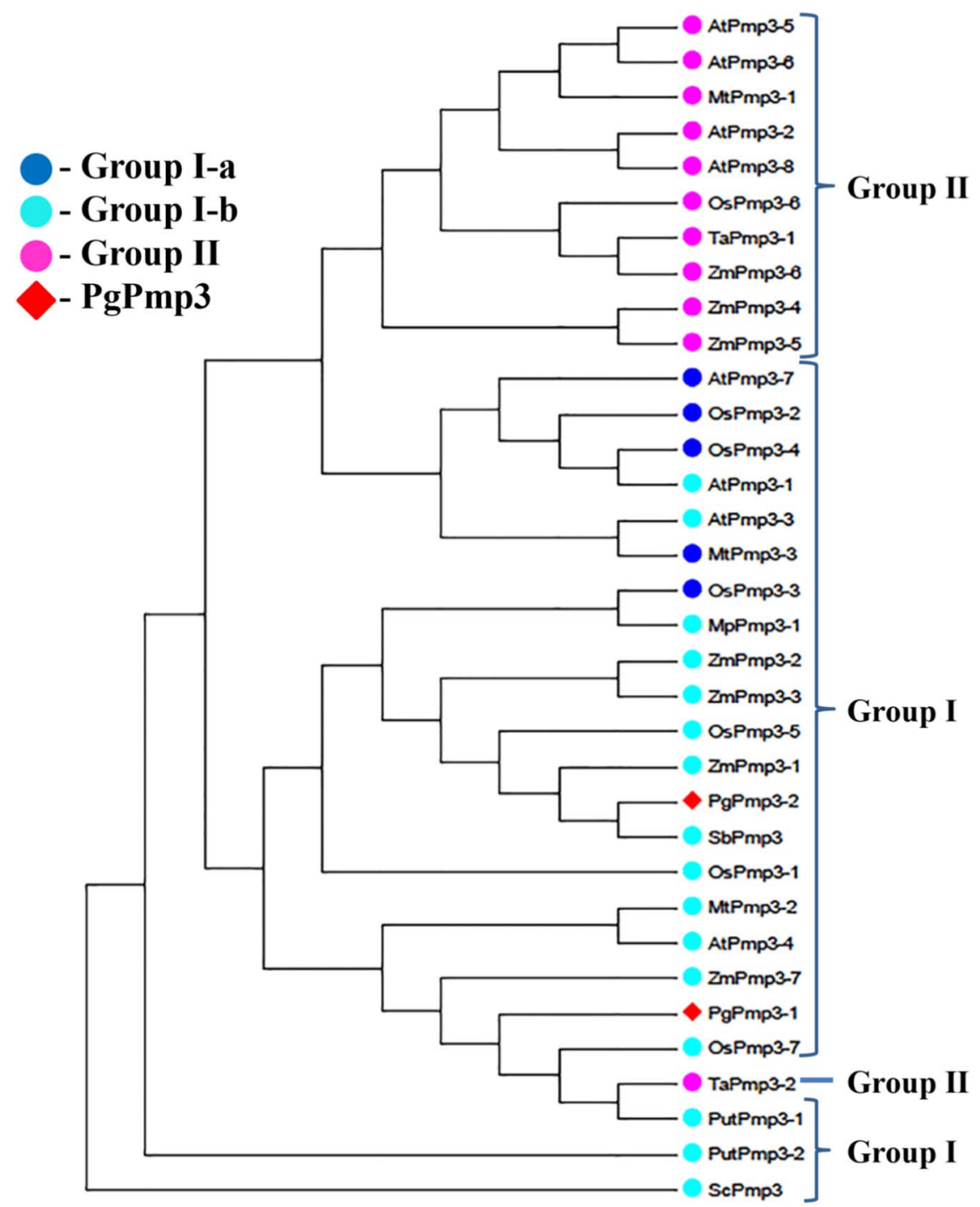

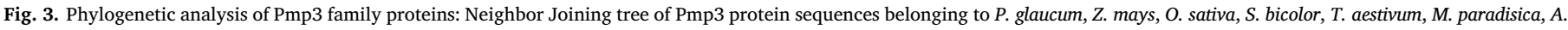

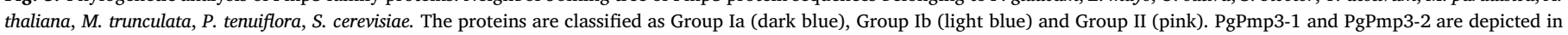
red. (For interpretation of the references to colour in this figure legend, the reader is referred to the web version of this article.)

PgPmp3-2, were selected from the following plants: $Z$. mays (NP_001107634.1, NP_001147403.2, NP_001147508.1, NP_001151727.2, NP_001151840.1, NP_001151922.1, NP_001152565.1, NP_001307385.1), O. sativa (AAG46140.1, AAT77365.1, XP_015633211.1, XP_015640253.1, XP_015643303.1, XP_015647973.1), Sorghum bicolor (XP_002440557.1), T. aestivum (AAN06944.1, CDM82662.1), Brachypodium distachyon (XP_003568974.1), Musa paradisica (ACA66247.1), A. thaliana (NP_001323801.1, NP_176067.1, NP_179982.1, NP_187239.1, NP_187240.1, NP_194794.1, NP_194795.1, NP_565897.1, NP_974629.1), Medicago truncatula (XP_003610298.1, XP_003626132.1, XP_013451651.1, XP_013458588.1), P. tenuiflora (BAG54793.1, BAG54794.1) and S. cerevisiae (NP_010562.1) and were used to conduct multiple sequence alignment analysis. All Pmp3 sequences were observed to be highly conserved and could be classified into two groups, I and II (Figs. 2, 3). In general, Group I Pmp3s have been reported to be 49 to 58 amino acids long; while the group II was 64 to 76 amino acids in length. Group I Pmp3s were further divided into sub-groups Ia and Ib on the basis of their C-termini hydropathicity (Group Ia proteins present a hydrophobic C-termini while Group Ib proteins have hydrophilic C-termini ends. Group II proteins have an extra 20 to 30 highly charged residues at the $\mathrm{C}$-terminus tail).

For a systematic study on the evolutionary relationship between Pmp3 proteins from P. glaucum and other organisms, a NJ phylogenetic tree was constructed with 1000 bootstrap iterations, by aligning the sequences. The phylogenetic tree showed distinct clades representing group I and group II Pmp3 proteins. While, both PgPmp3-1 and PgPmp3-2 broadly fall in group I, PgPmp3-1 was found to be evolutionarily close to Pmp3 from maize (ZmPmp3-7) while PgPmp32 showed a close relationship with SbPmp3 (Fig. 3).

\subsection{Structure prediction and network analysis of PgPmp3 proteins}

The secondary structure predicted using PSIPRED suggested a presence of five helices with a $\beta$-strand between the second and third helix in PgPmp3-1 (Fig. S1a). The TMHMM profile indicated transmembrane localization between the $5^{\text {th }}$ and $27^{\text {th }}$ positions, followed by an intracellular region ( 28 to 31 amino acids), tailed by another transmembrane region ( $32^{\text {nd }}$ to $54^{\text {th }}$ positions), as shown in Fig. 4a. The secondary structure of PgPmp3-2 showed four helices and a $\beta$-sheet (Fig. S1b). PgPmp3-2 was partially localized in the plasma membrane, separating two transmembrane regions, the first between $5^{\text {th }}$ and $22^{\text {nd }}$ positions and the second between $32^{\text {nd }}$ and $54^{\text {th }}$ positions. PgPmp3-2 showed a longer extracellular region between $23^{\text {rd }}$ to $31^{\text {st }}$ residues, as described in Fig. $4 \mathrm{~b}$.

To gain some insight regarding the interactions of Pmp3 proteins with other functional partners, network analysis was performed using the 
a

\section{CYTOPLASMIC}

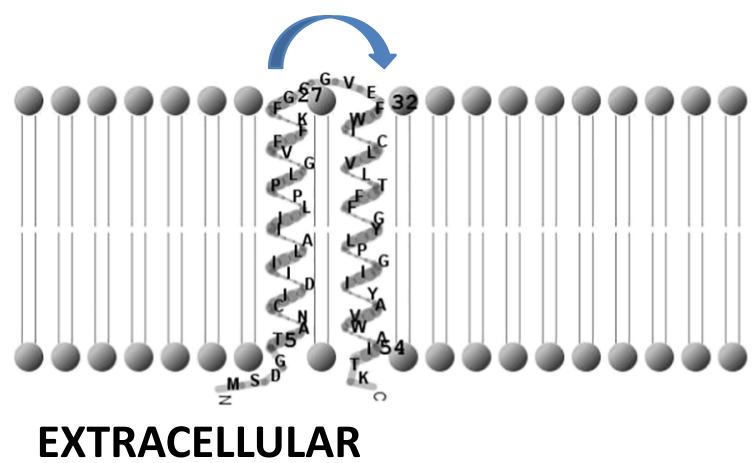

b

\section{EXTRACELLULAR}

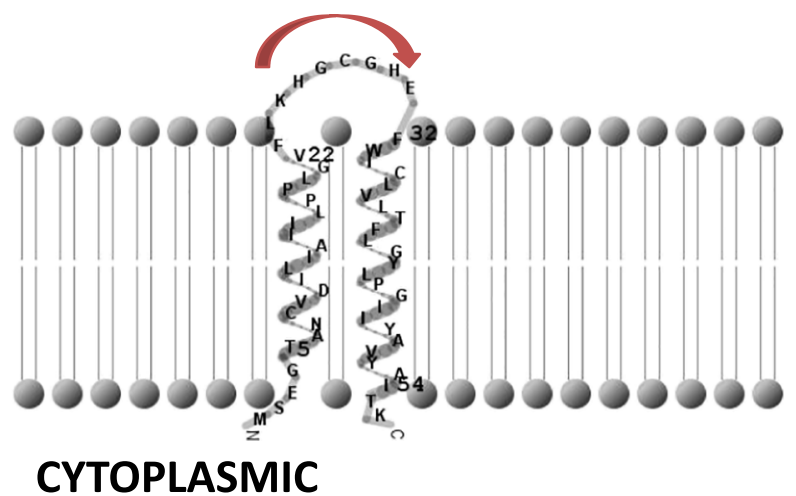

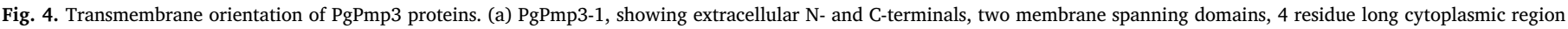
and (b) PgPmp3-2, showing cytoplasmic N- and C-terminals, two membrane spanning domains and a 9 residue long extracellular region.

STRING database. For this, LTI6A and LTI6B, homologs of PgPmp3-1 and PgPmp3-2 from Z. mays were used. Association of these two proteins with 8 functional partners with confidence score exceeding 0.4 were selected (Table 1). Proteins LTI6A and LTI6B exhibited co-occurrence across genomes. Proteins LTI6B and cl37957_1, homologs of PgPmp3-2 were predicted to be co-expressed along with gst30, dhn-2, gpm592 and GRMZM2G037452_P01 (Fig. 4b). Similarly, LTI6A and LTI6B were involved in protein binding and post-translational modifications with other proteins such as GRMZM2G181378_P01 (E3 ubiquitin-protein ligase) and GRMZM2G080439_P01 (uncharacterized protein) (Fig. 5a). The putative role of each of these proteins based on Gene Ontology and KEGG analyses could be linked to abiotic stress tolerance (Table 1) (Gene Ontology Consortium, 2015; Kanehisa et al., 2016).

\subsection{Expression profiling of PgPmp3 genes in response to abiotic stress treatments}

The expression of the Pmp3 genes from $P$. glaucum was analyzed in response to abiotic stress treatments such as low and high temperatures, salinity and drought. It was found that the expression of PgPmp3-1 and PgPmp3-2 genes was induced in response to cold stress showing maximum expression at $6 \mathrm{~h}$ and $8 \mathrm{~h}$, showing 5 -fold and 18-fold upregulation respectively (Fig. 6a). High temperature stress caused a rapid 4-fold and 48-fold higher upregulation up to $2 \mathrm{~h}$, followed by gradual downregulation in both PgPmp3-1 and PgPmp3-2, respectively
(Fig. 6b). Furthermore, the relative expression of both the genes showed gradual upregulation when subjected to salt stress and positively correlated with the duration of the stress (Fig. 6d). However, in plants subjected to drought stress, the relative transcript abundance of PgPmp3-1 decreased from 1.2 to 0.2 fold, over a period of $72 \mathrm{~h}$, whereas, PgPmp3-2 was steadily upregulated from 2 to 22 fold with time (Fig. 6c). Tukey-Kramer test revealed significant relative expression $(p<0.05)$ of PgPmp3-1 and PgPmp3-2 genes, at different time points in response to abiotic stress conditions (Fig. 6).

\section{Discussion}

P. glaucum (L.), commonly known as pearl millet belongs to the Poaceae family. It is mostly cultivated in the semi-arid regions, and is well adapted to heat, salinity and drought stress. Besides these stresses, $P$. glaucum also thrives in low temperatures (Desai et al., 2006) being treated as a post monsoon cool season crop in certain parts of India, growing in temperatures as low as 10 to $15^{\circ} \mathrm{C}$ (Mula et al., 2009). Though the crop thrives under low temperatures, poor seed set clubbed with low yields are main concern in certain genotypes. To counter such losses, it is important to elucidate the factors contributing to low temperature tolerance in $P$. glaucum genotypes. Abiotic stress conditions, particularly low temperature stress, affect the plasma membrane (Osakabe et al., 2013). Freezing temperatures are detrimental for the integrity of the membrane and may induce electrolyte leakages (Lyons,

Table 1

Predicted functional partners of Pmp3 proteins and interacting proteins as predicted using the search tool for the Retrieval of Interacting Genes/Proteins (STRING).

\begin{tabular}{|c|c|c|c|c|}
\hline String ID & Protein & Family & $\begin{array}{l}\text { Length (amino } \\
\text { acids) }\end{array}$ & Function \\
\hline umc1359 & LTI6B & Pmp3 & 57 & $\begin{array}{l}\text { Membrane potential modulator and resistance to ionic } \\
\text { stress }\end{array}$ \\
\hline cl37957_1 & uncharacterized protein & Pmp3 & 58 & $\begin{array}{l}\text { Membrane potential modulator and resistance to ionic } \\
\text { stress }\end{array}$ \\
\hline pmpm3 & LTI6B & Pmp3 & 75 & $\begin{array}{l}\text { Membrane potential modulator and resistance to ionic } \\
\text { stress }\end{array}$ \\
\hline pmpm5 & LTI6A & Pmp3 & 56 & $\begin{array}{l}\text { Membrane potential modulator and resistance to ionic } \\
\text { stress }\end{array}$ \\
\hline gst30 & Glutathione-S-transferase 30 & Glutathione-S-transferase 30 & 257 & Detoxification of electrophilic compounds \\
\hline GRMZM2G181378_P01 & uncharacterized protein & E3 ubiquitin-protein ligase & 871 & Regulation of cell processes \\
\hline GRMZM2G080439_P01 & uncharacterized protein & E3 ubiquitin-protein ligase & 880 & Regulation of cell processes \\
\hline dhn-2 & dehydrin & dehydrin & 289 & Survival during drought stress \\
\hline GRMZM2G037452_P01 & uncharacterized protein & $\begin{array}{l}\text { CYSTM (cysteine-rich membrane } \\
\text { proteins) }\end{array}$ & 69 & Cell signaling \\
\hline
\end{tabular}


a

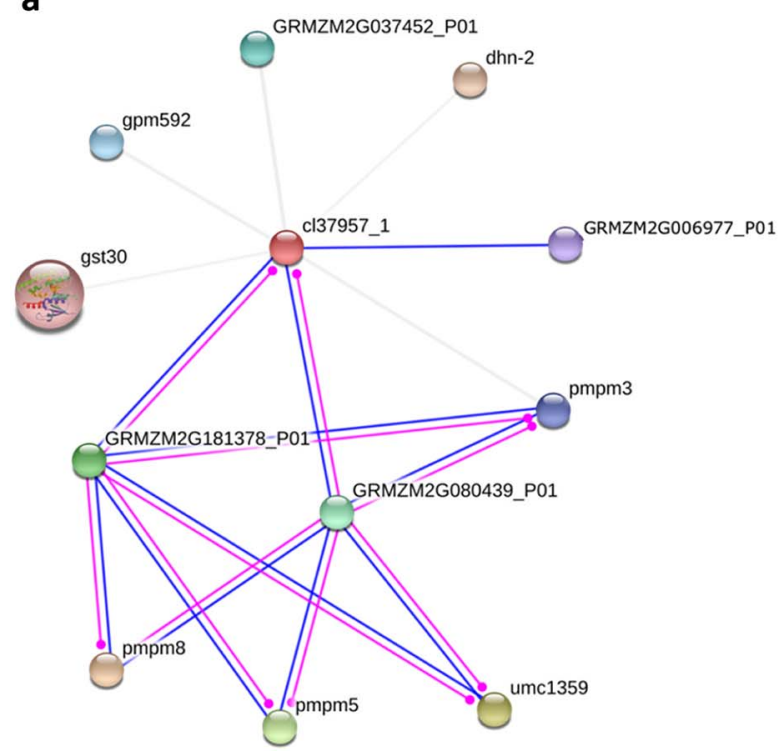

b

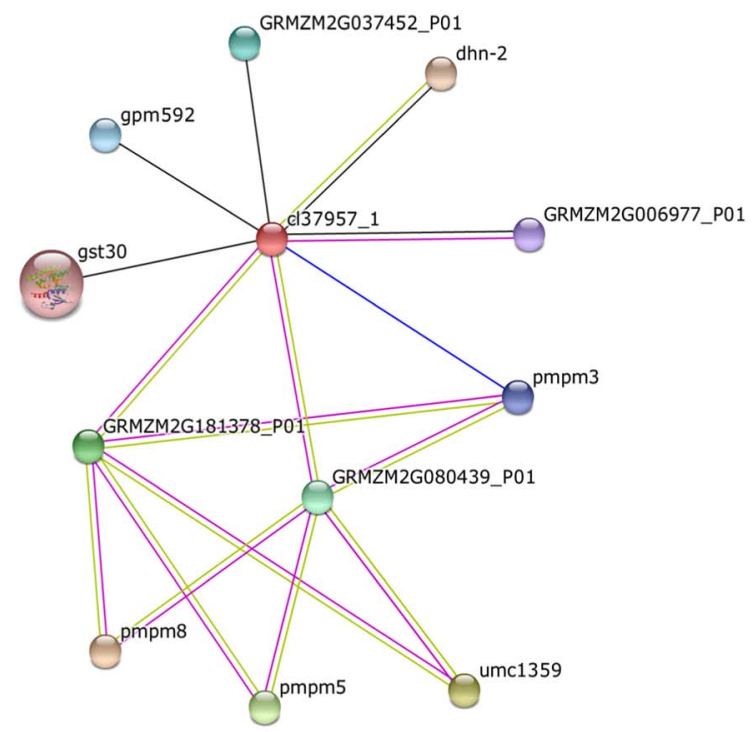

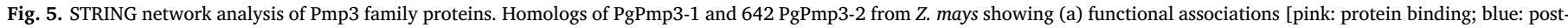

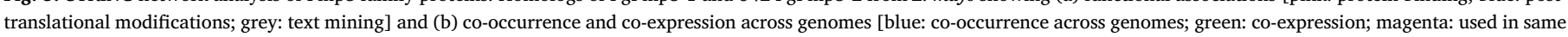
experiments]. (For interpretation of the references to color in this figure legend, the reader is referred to the web version of this article.)

2012). Temperatures as low as $4{ }^{\circ} \mathrm{C}$ affect the ion transport at the membrane. Many integral and surface proteins are known to contribute towards maintaining membrane integrity during stress conditions. For example, $R C I 2 A$ and $R C I 2 B$ gene homologs of Pmp3 from A. thaliana showed expression in response to cold, salt and dehydration stresses (Medina et al., 2001). Similarly, the expression of RCI2 gene, another homolog of Pmp3 from M. paradisiaca, conferred enhanced tolerance to low temperatures when expressed in tobacco (Feng et al., 2009). Likewise, Pmp3 genes from various organisms are reported to be expressing under various abiotic stress conditions, indicating that these might play a crucial role in stress tolerance.

In the present study, two genes PgPmp3-1 and PgPmp3-2 from $P$.

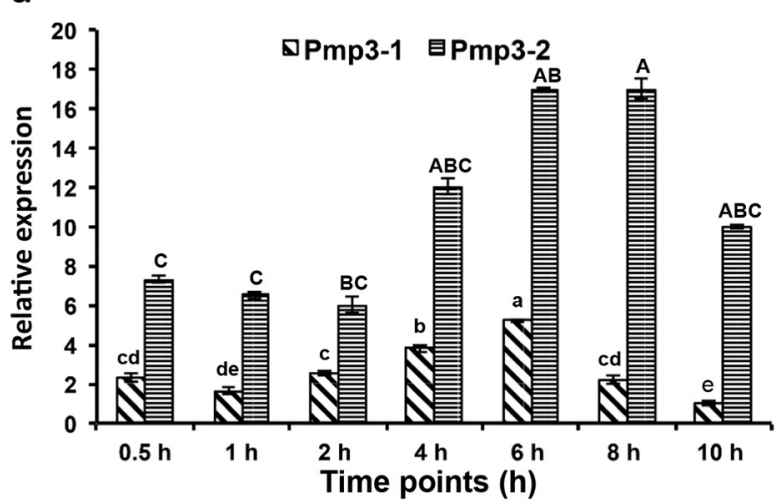

C

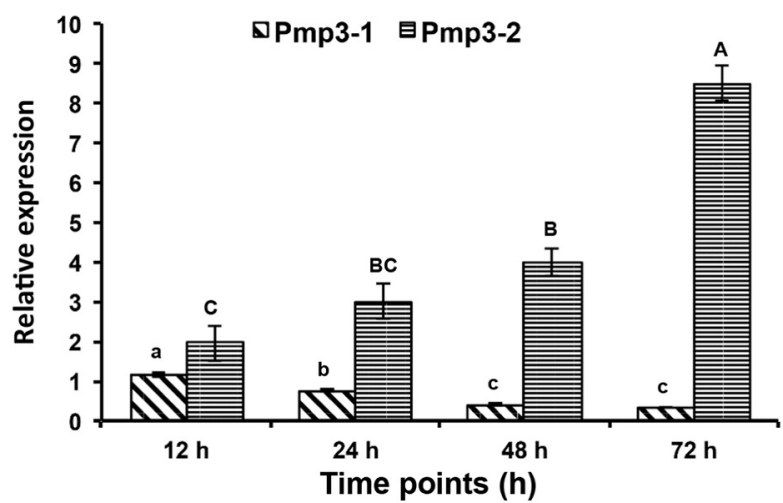

b

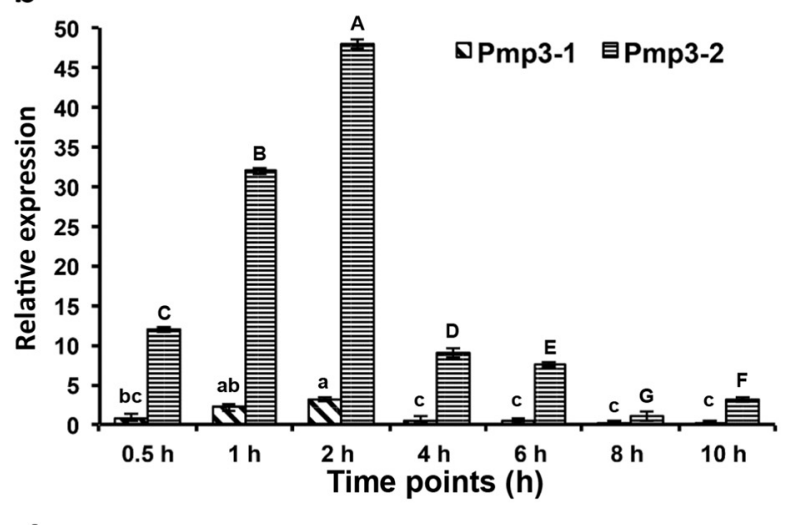

d

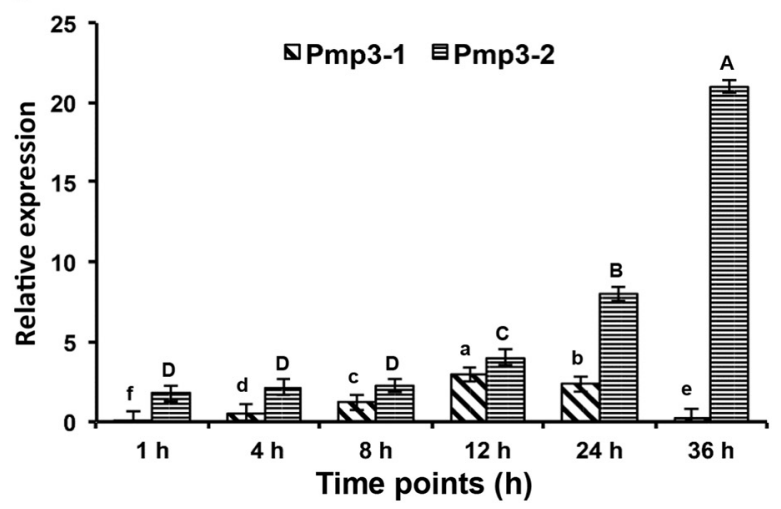

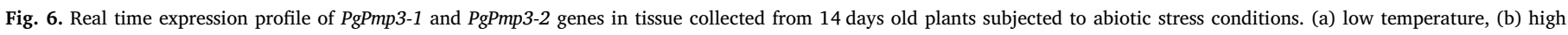

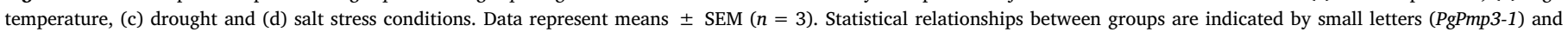
capital letters (PgPmp3-2) where significant differences were detected $(p<0.05)$. 
glaucum were identified based on their high similarity with the yeast Pmp3 genes. These genes were isolated and cloned from the subtracted stress responsive cDNA library, prepared in our earlier efforts (Mishra et al., 2007). Our current study reveals that PgPmp3-1 had one intron while PgPmp3-2 gene has none (Fig. 1). These observations are in agreement with previous report on Pmp3/RCI genes that have less number of introns, and are expressed under stress conditions (Zhao et al., 2014). Low intron number has also been reported in other stress responsive genes such as trehalose-6-phosphate synthase and late embryogenesis abundant (LEA) (Liang et al., 2016), possibly associated with enhanced gene expression by improving the efficiency of transcript production.

PgPmp3-1 and PgPmp3-2 showed homology with genes encoding Pmp3/RCI2/LTI and hydrophobic proteins from other organisms. PgPmp3-1 showed 98\% identity with its closest homolog from Z. mays and $96 \%$ identity with its homolog from S. bicolor, while PgPmp3-2 showed 94\% identity with $S$. bicolor Pmp3 (Fig. 2), indicating evolutionarily conservation in genes of major cereal crop grasses. The structural diversity of Pmp3 proteins that is reflected from the multiple sequence alignment study is in agreement with previous reports by Medina et al. (2007) and Rocha (2016). Comparative analyses indicated that both genes belonged to the sub-group Ib, as these were predicted to be of 56 amino acids length with hydrophilic C-termini. This structural diversity was also evident from the phylogenetic analysis (Fig. 3). The NJ tree exhibited different clades corresponding to the Pmp3 groups - Ia, Ib and II. The phylogenetic groupings closely matched the hydropathicity of their C-termini.

The PgPmp3-1 and PgPmp3-2 proteins were predicted to contain two hydrophobic stretches corresponding to two transmembrane domains. The orientation of PgPmp3-1 and PgPmp3-2 in the plasma membrane varied with PgPmp3-1 showing extracellular C- and N-terminals as opposed to PgPmp3-2, which had cytoplasmic ends (Fig. 4a and b). The amino acids Phe, Phe and Val at the 24th, 26th and 30th positions in PgPmp3-1 were replaced by Leu, His and His respectively in PgPmp3-2 (Fig. 2a). It is believed that the orientation of the membrane proteins depends on the amino acid composition of the cytoplasmic and extracellular sides (Nakashima and Nishikawa, 1992). Thus, the histidine residues in PgPmp3-2 might be responsible for extracellular orientation of the region. Furthermore, according to ProtLoc (http://bioinf.uab.es/ cgi-bin/trsdb/protloc.cgi), the algorithm described by Cedano et al. (1997), the peptide fragment FLKHGCGHE from PgPmp3-2 showed a likeliness of extracellular localization, whereas the fragment CGVEF from PgPmp3-1 was predicted to have intracellular localization. Although the use of structure prediction tools shed a light upon the structure and localization of PgPmp3 proteins, little is known about the exact topology of these proteins through experimental studies. Earlier, Rocha (2016) obtained conflicting topology results using various prediction tools and hence highlighted limitations in such studies. Considering this, in our study we chose to use multiple prediction tools such as TMHMM, MEMSTAT, Phobius, Philius and Scampi to avoid such conflicts. The topology of PgPmp3-1 predicted by TMHMM was in agreement with results of MEMSTAT, while the TMHMM topology of PgPmp3-2 matched the output of tools such as Phobius, Philius and Scampi. The presence of conserved domains related to the proteolipid membrane potential modulator, reflects the role of Pmp3 in abiotic stress tolerance, especially under cold stress (Shabala et al., 2016). Moreover, the Gene Ontology server suggested that Pmp3 proteins might mediate a proton leak suggesting that Pmp3 plays a role in ion homeostasis, an important step in countering salt stress (Zhu, 2001).

Proteins homologous to the Pmp3 family, that is, LTI6A and LTI6B from $Z$. mays, were analyzed in silico for presence of functional interactions with other orthologs by using STRING database. This study revealed the synergistic interaction of proteins contributing towards abiotic stress tolerance. The proteins were co-expressed with other Pmp3 proteins, responsible for membrane potential modulation, particularly to resist ionic stress (Fig. 5a and b; Table 1). Other proteins such as dehydrin, glutathione-s-transferase 30 (gst30), ubiquitin-protein transferase and cysteine-rich membrane proteins (CYSTM) were found to be associated with LTI6A and LTI6B. Dehydrin, a protein aiding survival during drought stress (Graether and Boddington, 2014) is highly hydrophilic, and binds to the membrane periphery playing an important role in protecting the membrane from freezing (Drira et al., 2013). Likewise, glutathione-s-transferase involved in detoxification of electrophilic compounds, has been shown to bestow salt tolerance to the cell (Chen et al., 2012). In a similar fashion, ubiquitin-protein transferase, a protein involved in ubiquitination has been associated with Pmp3 proteins in stress tolerance. Ubiquitination mediated by E3 ubiquitin ligases regulate numerous cell processes and facilitate tolerance towards stress conditions (Lyzenga and Stone, 2012). Similarly, cysteine rich membrane proteins, particularly receptor-like kinases, activate signaling pathways in response to environmental stimuli (Kanehisa et al., 2016). As a whole, the combined effects of all these associations potentially aid the plant in building tolerance to adverse environmental conditions.

It is a well-known fact that abiotic stresses such as salt, low temperature and drought conditions result in ionic imbalance leading to secondary stresses (Mahajan and Tuteja, 2005). The immediate defense response of plants involves stress alleviation, followed by maintaining homeostasis and regaining growth. This requires complex molecular responses (Zhu et al., 1997). In our study, the $P$. glaucum genes belonging to the Pmp3 family were found to show increased expression under simulated low temperature and high salt stress (Fig. 6a and d). In addition to cold and salinity, PgPmp3-2 was also induced by drought conditions (Fig. 6c). This could be attributed to presence of different cis-regulatory elements in the promoter region of PgPmp3-1 and PgPmp3-2 genes. However, this specifically could not be studied in detail due to nonavailability of $P$. glaucum genome sequence. In several studies, Pmp3 genes from various sources have demonstrated an increase in transcript levels in response to cold and salinity. For instance, expression of a number of Pmp3 genes from $A$. thaliana and $Z$. mays has been reported to be induced in response to cold, drought and salinity stress (Rocha, 2016). Furthermore, maize Pmp3 genes were also reported to be involved in salt stress tolerance (Fu et al., 2012). Similarly, Pmp3 from the yeast (S. cerevisiae) has not only shown to be expressed in response to abiotic stress conditions such as low temperature and salt stress (Bari et al., 2015), but also deletions in Pmp3 have led to membrane hyperpolarization and salt sensitivity (Navarre and Goffeau, 2000). In another study, P. tenuiflora Pmp3 genes, along with their rice homologs functioned in reversing stress induced membrane hyperpolarization and countering salt stress (Chang-Qing et al., 2008).

\section{Conclusion}

In this study, we have cloned and characterized two $P$. glaucum genes belonging to the Pmp3 family, encoding 56 amino acid long transmembrane proteins. These genes were found to share major identity with stress inducible genes belonging to other cereals such as maize and sorghum. Conserved domains corresponding to a plasma membrane potential modulator were identified in these proteins, supporting their role in abiotic stress response. PgPmp3-1 and PgPmp3-2 were induced under abiotic stress conditions such as high salt and low temperature. PgPmp3-2 also showed enhanced expression under drought stress. Moreover, Pmp3 proteins were predicted to interact along with other proteins contributing to stress tolerance in plants. Further studies pertaining to the mechanism of Pmp3 in stress alleviation may lead to greater insight in plant stress tolerance.

Supplementary data to this article can be found online at http://dx. doi.org/10.1016/j.plgene.2017.05.002.

\section{Author contribution statement}

Conceived and designed the experiments: PSR and MKR. Performed the experiments: RY, RN and PSR Analyzed the data: RY, TC and PBM. Wrote the paper: RY, RN and PSR. 


\section{Conflict of interest}

The authors declare no conflict of interest.

\section{Acknowledgements}

This work was supported partially by the Department of Biotechnology, Ministry of Science and Technology (Government of India) to MKR. PSR acknowledges the Department of Science and Technology (DST), Govt. of India for financial support through INSPIRE Faculty Program (Award No. IFA-LSPA-06) and Young Scientist Scheme (SB/YS/LS-12/2013).

\section{References}

Arazi, T., Sunkar, R., Kaplan, B., Fromm, H., 1999. A tobacco plasma membrane calmodulin-binding transporter confers $\mathrm{Ni}^{2+}$ tolerance and $\mathrm{Pb}^{2+}$ hypersensitivity in transgenic plants. Plant J. 20, 171-182.

Bari, V.K., Sharma, S., Alfatah, M., Mondal, A.K., Ganesan, K., 2015. Plasma membrane proteolipid 3 protein modulates amphotericin b resistance through sphingolipid biosynthetic pathway. Sci. Rep. 5, 9685.

Buchan, D.W., Minneci, F., Nugent, T.C., Bryson, K., Jones, D.T., 2013. Scalable web services for the PSIPRED protein analysis workbench. Nucleic Acids Res. 41, W349-W357.

Cedano, J., Aloy, P., Perez-Pons, J.A., Querol, E., 1997. Relation between amino acid composition and cellular location of proteins. J. Mol. Biol. 266, 594-600.

Chang-Qing, Z., Shunsaku, N., Shenkui, L., Tetsuo, T., 2008. Characterization of two plasma membrane protein 3 genes (PutPMP3) from the alkali grass Puccinellia tenuiflora and functional comparison of the rice homologues OsLti6a/b from rice. BMB Rep. 41, $448-454$

Chen, J.H., Jiang, H.W., Hsieh, E.J., Chen, H.Y., Chien, C.T., Hsieh, H.L., Lin, T.P., 2012. Drought and salt stress tolerance of an Arabidopsis glutathione S-transferase U17 knockout mutant are attributed to the combined effect of glutathione and abscisic acid. Plant Physiol. 158, 340-351.

De Block, J., Szopinska, A., Guerriat, B., Dodzian, J., Villers, J., Hochstenbach, J.F., Morsomme, P., 2015. Yeast Pmp3p has an important role in plasma membrane organization. J. Cell Sci. 128, 3646-3659.

Desai, M.K., Mishra, R.N., Verma, D., Nair, S., Sopory, S.K., Reddy, M.K., 2006. Structural and functional analysis of a salt stress inducible gene encoding voltage dependent anion channel (VDAC) from pearl millet (Pennisetum glaucum). Plant Physiol. Biochem. 44, 483-493.

Drira, M., Saibi, W., Brini, F., Gargouri, A., Masmoudi, K., Hanin, M., 2013. The Ksegments of the wheat dehydrin DHN-5 are essential for the protection of lactate dehydrogenase and beta-glucosidase activities in vitro. Mol. Biotechnol. 54, 643-650.

Feng, D.R., Liu, B., Li, W.Y., He, Y.M., Qi, K.B., Wang, H.B., Wang, J.F., 2009. Overexpression of a cold-induced plasma membrane protein gene (MpRCI) from plantain enhances low temperature-resistance in transgenic tobacco. Environ. Exp. Bot. 65, 395-402.

Fu, J., Zhang, D.F., Liu, Y.H., Ying, S., Shi, Y.S., Song, Y.C., Li, Y., Wang, T.Y., 2012 Isolation and characterization of maize PMP3 genes involved in salt stress tolerance. PLoS One 7, e31101.

Gene Ontology Consortium, 2015. Gene Ontology Consortium: going forward. Nucleic Acids Res. 43, D1049-D1056.

Goddard, N.J., Dunn, M.A., Zhang, L., White, A.J., Jack, P.L., Hughes, M.A., 1993. Molecular analysis and spatial expression pattern of a low-temperature-specific barley gene, blt101. Plant Mol. Biol. 23 (4), 871-879.

Graether, S.P., Boddington, K.F., 2014. Disorder and function: a review of the dehydrin protein family. Front. Plant Sci. 5, 576.

Islam, S.M., Tuteja, N., 2012. Enhancement of androgenesis by abiotic stress and other pretreatments in major crop species. Plant Sci. 182, 134-144.

Kanehisa, M., Sato, Y., Kawashima, M., Furumichi, M., Tanabe, M., 2016. KEGG as a reference resource for gene and protein annotation. Nucleic Acids Res. 44 D457-D462.

Khurana, N., Chauhan, H., Khurana, P., 2015. Characterization of a chloroplast localized wheat membrane protein (TaRCI) and its role in heat, drought and salinity stress tolerance in Arabidopsis thaliana. Plant Gene 4, 45-54.

Liang, Y., Xiong, Z., Zheng, J., Xu, D., Zhu, Z., Xiang, J., Gan, J., Raboanatahiry, N., Yin, Y., Li, M., 2016. Genome-wide identification, structural analysis and new insights into late embryogenesis abundant (LEA) gene family formation pattern in Brassica napus. Sci. Rep. 6.

Li, J., Yu, G., Sun, X., Liu, Y., Liu, J., Zhang, X., Jia, C., Pan, H., 2015. AcPIP2 a plasma membrane intrinsic protein from halophyte Atriplex canescens enhances plant growth rate and abiotic stress tolerance when overexpressed in Arabidopsis thaliana. Plant Cell Rep. 34, 1401-1415.
Lyons, J., 2012. (Ed) low Temperature Stress in Crop Plants: The Role of the Membrane Elsevier.

Lyzenga, W.J., Stone, S.L., 2012. Abiotic stress tolerance mediated by protein ubiquitination. J. Exp. Bot. 63, 599-616.

Mahajan, S., Tuteja, N., 2005. Cold, salinity and drought stresses: an overview. Arch. Biochem. Biophys. 444, 139-158.

Marchler-Bauer, A., Lu, S., Anderson, J.B., Chitsaz, F., Derbyshire, M.K., DeWeese-Scott, C., Fong, J.H., Geer, L.Y., Geer, R.C., Gonzales, N.R., Gwadz, M., Hurwitz, D.I., Jackson, J.D., Ke, Z., Lanczycki, C.J., Lu, F., Marchler, G.H., Mullokandov, M., Omelchenko, M.V., Robertson, C.L., Song, J.S., Thanki, N., Yamashita, R.A., Zhang, D., Zhang, N., Zheng, C., Bryant, S.H., 2011. CDD:a conserved domain database for the functional annotation of proteins. Nucleic Acids Res. 39, D225-D229.

Marmagne, A., Rouet, M.A., Ferro, M., Rolland, N., Alcon, C., Joyard, J., Garin, J., Barbier-Brygoo, H., Ephritikhine, G., 2004. Identification of new intrinsic proteins in Arabidopsis plasma membrane proteome. Mol. Cell. Proteomics 3, 675-691.

Medina, J., Catala, R., Salinas, J., 2001. Developmental and stress regulation of RCI2A and RCI2B two cold-inducible genes of Arabidopsis encoding highly conserved hydrophobic proteins. Plant Physiol. 125, 1655-1666.

Medina, J., Ballesteros, M.L., Salinas, J., 2007. Phylogenetic and functional analysis of Arabidopsis RCI2 genes. J. Exp. Bot. 58, 4333-4346.

Mishra, R.N., Reddy, P.S., Nair, S., Markandeya, G., Reddy, A.R., Sopory, S.K., Reddy, M.K., 2007. Isolation and characterization of expressed sequence tags (ESTs) from subtracted cDNA libraries of Pennisetum glaucum seedlings. Plant Mol. Biol. 64, 713-732.

Mula, R., Rai, K.N., Dangaria, C.J., Kulkarni, M.P., 2009. Pearl millet as a postrainy cool season crop: case studies from Gujarat and Maharashtra, India. J. SAT Agric. Res. 7, 445-458.

Nakashima, H., Nishikawa, K., 1992. The amino acid composition is different between the cytoplasmic and extracellular sides in membrane proteins. FEBS Lett. 303, 141-146.

Navarre, C., Goffeau, A., 2000. Membrane hyperpolarization and salt sensitivity induced by deletion of PMP3 a highly conserved small protein of yeast plasma membrane. EMBO J. 19, 2515-2524.

Osakabe, Y., Mizuno, S., Tanaka, H., Maruyama, K., Osakabe, K., Todaka, D., Fujita, Y., Kobayashi, M., Shinozaki, K., Yamaguchi-Shinozaki, K., 2010. Overproduction of the membrane-bound receptor-like protein kinase 1 RPK1 enhances abiotic stress tolerance in Arabidopsis. J. Biol. Chem. 285, 9190-9201.

Osakabe, Y., Yamaguchi-Shinozaki, K., Shinozaki, K., Tran, L.S., 2013. Sensing the environment: key roles of membrane-localized kinases in plant perception and response to abiotic stress. J. Exp. Bot. 64, 445-458.

Panjabi-Sabharwal, V., Karan, R., Khan, T., Pareek, A., 2010. Abiotic stress adaptation in plants. In: Pareek, A., Sopory, S.K., Bohnert, H.J., Govindjee (Eds.), Physiological Molecular and Genomic Foundation. Springer, Dordrecht the Netherlands, pp. $177-198$.

Pfaffl, M.W., Horgan, G.W., Dempfle, L., 2002. Relative expression software tool (REST) for group-wise comparison and statistical analysis of relative expression results in real-time PCR. Nucleic Acids Res. 30, e36.

Reddy, P.S., Reddy, G.M., Pandey, P., Chandrasekhar, K., Reddy, M.K., 2012. Cloning and molecular characterization of a gene encoding late embryogenesis abundant protein from Pennisetum glaucum: protection against abiotic stresses. Mol. Biol. Rep. 39, 7163-7174.

Reddy, P.S., Kavi Kishor, P.B., Seiler, C., Kuhlmann, M., Eschen-Lippold, L., Lee, J., Reddy, M.K., Sreenivasulu, N., 2014. Unraveling regulation of the small heat shock proteins by the heat shock factor HvHsfB2c in barley: its implications in drought stress response and seed development. PLoS One 9, e89125.

Reddy, P.S., Sharma, K.K., Vadez, V., Reddy, M.K., 2015. Molecular cloning and differential expression of cytosolic class I small hsp gene family in Pennisetum glaucum (L.). Appl. Biochem. Biotechnol. 176, 598-612.

Rocha, P.S., 2016. Plant abiotic stress-related RCI2/PMP3s: multigenes for multiple roles. Planta 243, 1-12.

Roy, P., Niyogi, K., SenGupta, D.N., Ghosh, B., 2005. Spermidine treatment to rice seedlings recovers salinity stress-induced damage of plasma membrane and PMbound $\mathrm{H}+$-ATPase in salt-tolerant and salt-sensitive rice cultivars. Plant Sci. 168, 583-591.

Serrano, R., Rodriguez-Navarro, A., 2001. Ion homeostasis during salt stress in plants. Curr. Opin. Cell Biol. 13, 399-404.

Shabala, S., Bose, J., Fuglsang, A.T., Pottosin, I., 2016. On a quest for stress tolerance genes: membrane transporters in sensing and adapting to hostile soils. J. Exp. Bot. 67 1015-1031.

Szklarczyk, D., Franceschini, A., Wyder, S., Forslund, K., Heller, D., Huerta-Cepas, J., Simonovic, M., Roth, A., Santos, A., Tsafou, K.P., Kuhn, M., Bork, P., Jensen, L.J., von Mering, C., 2015. STRING v10:protein-protein interaction networks integrated over the tree of life. Nucleic Acids Res. 43, D447-D452.

Zhao, Y., Tong, H., Cai, R., Peng, X., Li, X., Gan, D., Zhu, S., 2014. Identification and characterization of the RCI2 gene family in maize (Zea mays). J. Genet. 93, 655-666.

Zhu, J.K., 2001. Plant salt tolerance. Trends Plant Sci. 6, 66-71.

Zhu, J.K., Hasegawa, P.M., Bressan, R.A., 1997. Molecular aspects of osmotic stress in plants. Crit. Rev. Plant Sci. 16, 253-277. 